MATEC Web of Conferences 32, 03003 (2015)

DOI: $10.1051 /$ matecconf $/ 20153203003$

O Owned by the authors, published by EDP Sciences, 2015

\title{
On-Machine Measurement of Cutting Tool Edge Profile by Detecting Fluorescence from Cutting Fluid
}

\author{
Kenji Maruno ${ }^{1,}$, Masaki Michihata $^{2}$, Yasuhiro Mizutani ${ }^{1}$, and Yasuhiro Takaya ${ }^{1}$ \\ ${ }^{1}$ Department of Mechanical Engineering, Osaka University, Japan \\ ${ }^{2}$ Research Center of Advanced Science and Technology (RCAST), The University of Tokyo, Japan
}

\begin{abstract}
We propose a novel on-machine measurement method of the tool cutting edge profile by using cutting fluid adhered on the tool surface. In an environment of on-machine tool profile measurement, the profile is difficult to measure with the conventional optical method since a cutting fluid adhered on the tool surface interferes the measurement. To overcome this problem, we propose the profile measurement method by confocal fluorescent detection from the cutting fluid adhered the tool surface. To verify the fundamental validity of the principle, fluorescence from horizontal and vertical surface were detected with a developped fluorescent confocal microscope by using fluorescent dye and silicon wafer. Furthermore, a cutting edge profile of horizontal plane of milling tool with wear was measured with the proposed method using the fluorescent dye as the basic verification. From this result, it is found that the proposed method enables to measure the tool wear at the surface with the oil adhering slightly, however the measurement capability of the measurement might depend on the thickness of the oil
\end{abstract}

\section{Introduction}

The profile of cutting tool edge has a great influence on the performance of cutting. In general, the shape of cutting tool edge is deformed to chipping and wear, etc., by the repeated processing and the deformation affects directly on the surface of work-piece. Thus, monitoring the shape of the cutting tool edge is demanded for further high accuracy machining. Previously, it was possible to observe the profile in out of the machine environment by using Scanning Electron Microscope (SEM) or profile meter [1]. However, tool profile measurement by removing the tool from the machine, has some problems such as a long measurement time, and a positioning error that occurs upon re-attachment of the tool. In order to solve those problems, the on-machine measuring technique is demanded. Although the measurement by applying the AFM [2] or an optical method [3, 4] have been proposed as on-machine measurement technology so far, those use are limited and lack versatility.

Therefore, we propose the novel on-machine cutting tool edge profile measurement method by using fluorescent detection from cutting fluid adhering to the tool surface. The proposing method has feasibility as onmachine measuring method of the cutting tool edge since having 5 following advantages :

1. High-speed and non-contact measurement

2. Appropriate method for on-machine measurement since using cutting fluid adhered to the surface during machining

\footnotetext{
${ }^{\text {a }}$ Corresponding author: maruno@optim.mech.eng.osaka-u.ac.jp
}

3. Possible to measure vertical or more inclined surface of complex edge shape of cutting tool

4. Possible to measure without speckle noise affection by excitation laser for detecting incoherent fluorescence

5. The presence of cutting fluid with a fluorescent properties have been many reports [5], having a wide application range for on-machine measurement

In this paper, the principle of the measurement method of the tool cutting edge profile by fluorescence detection from cutting fluid is described. As a basic experiment, detections for horizontal and vertical surfaces with the proposed method were implemented, by using immersion oil dissolving a fluorescent dye, instead of cutting fluid. Furthermore, a horizontal surface profile of cutting tool was measured, by detecting fluorescence from immersion oil dissolving a fluorescent dye. From those results, discussion about the selection of excitation light spectra for establishing the proposed method was given.

\section{Principle of cutting tool edge profile measurement by detecting fluorescence from cutting fluid}

As shown in Fig. 1, the focused spot of the excitation light of the optical system passes the tool surface covered with cutting fluid that has fluorescent property. When the focused spot illuminates cutting fluid that covers the surface, the fluorescence from the cutting fluid excited by 
the excitation light is returned to an objective lens and detected by the optical system as a fluorescent signal. And then the spot of the excitation light subsequently passed through the area of the cutting fluid and reaches the tool surface, the detected fluorescence signal is decreased rapidly. By detecting the peak position of the fluorescence signal in this way, the position of the tool surface can be identified. The profile of the cutting edge of a tool can be measured through the same measurements in different areas of the tool surface. It has been reported that fluorescence emits radially in uniform intensity without depending on the irradiation direction of the excitation light [6]. Therefore, detected signal is hard to be affected from the slope of the measured tool surface. Moreover, even when the focusing spot of the excitation light is irradiated to a vertical surface that has a slope of 90 degrees or more by the objective lens as shown in Fig.1, it is possible to detect the fluorescence and measure the surface. Since this measurement allows the signal detection by rotating or scanning the tool itself attached to the mother machine, the operating mechanism of the optical system itself is not required. Furthermore, by using the confocal optical system, it is possible to measure the tool surface coordinate in the sub-micron precision.

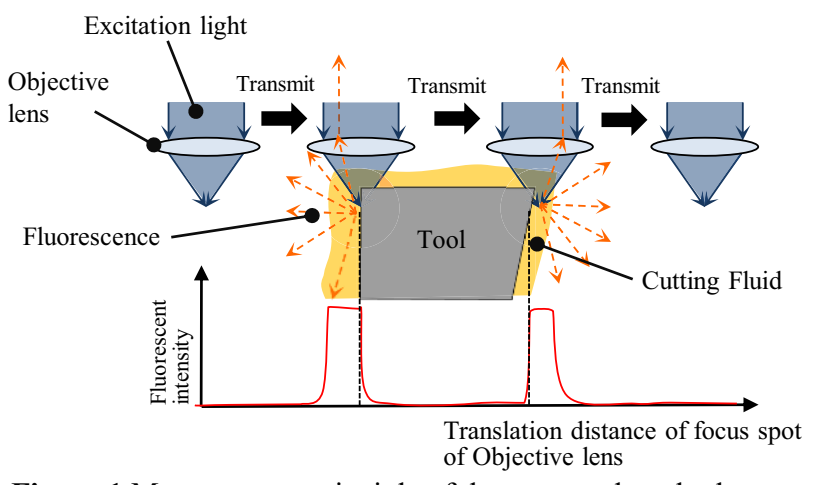

Figure 1 Measurement principle of the proposed method

\section{Experimental apparatus}

To verify the basic principle of the proposed method, a fluorescent confocal optical system is developped. Immersion oil dissolved a fluorescent dye that the optical properties are known (hereinafter, referred to as "Fluorescent oil") is used as substitution of cutting fluid.

Fig. 2 shows a schematic of optical system to detect fluorescence from Fluorescent oil. Diode laser $(\lambda=458$ $\mathrm{nm}$ ) for exciting fluorescence travels to the optical system through the optical fiber. The laser is collimated by the collimator lens. The Keplerian beam expander adjusts the beam to enter pupil of the objective lens. The specimen is positioned by using the 3 -axes stage that is, the focal spot was fixed and the specimen was displaced in this system. Exciting laser reaches in the fluorescent layer, and then fluorescence is collimated in the objective lens. The dichroic mirror allows fluorescence to pass to the pinhole. The fluorescence passes through long-pass filter which limits exciting laser, which reach to the detector behind the pinhole (Diameter $=20 \mu \mathrm{m})$. Fluorescence is weak, so Photo Multiplier Tube (PMT) is used as the detector. The
CCD camera is used as viewing system to observe the beam spot on specimen in order to adjust the optical arrangement. An image can be seen with setting mirror between tube lens and the CCD camera.

In this paper, Fluorescent oil dissolved fluorescent dye (International Science Technology, CO.LTD, Fluolid-WOrange) in immersion oil (Nikon CO.LTD, $\mathrm{n}=1.515$ ) is used as a fluorescent source in place of the cutting fluid. By substituting fluorescence from the fluorescent dye, it can be detected more stable and stronger fluorescent intensity than fluorescent detection from cutting fluid. Furthermore, since immersion oil used in Fluorescent oil is non-volatile, oil film thickness is not changed at the time of measurement. For these reasons, Fluorescent oil was chosen in the fundamental experiment. Figure 3 shows absorption and emission spectra of the fluorescent dye. Fluorescent oil is made by dissolving the fluorescent dye in the immersion oil until saturation.

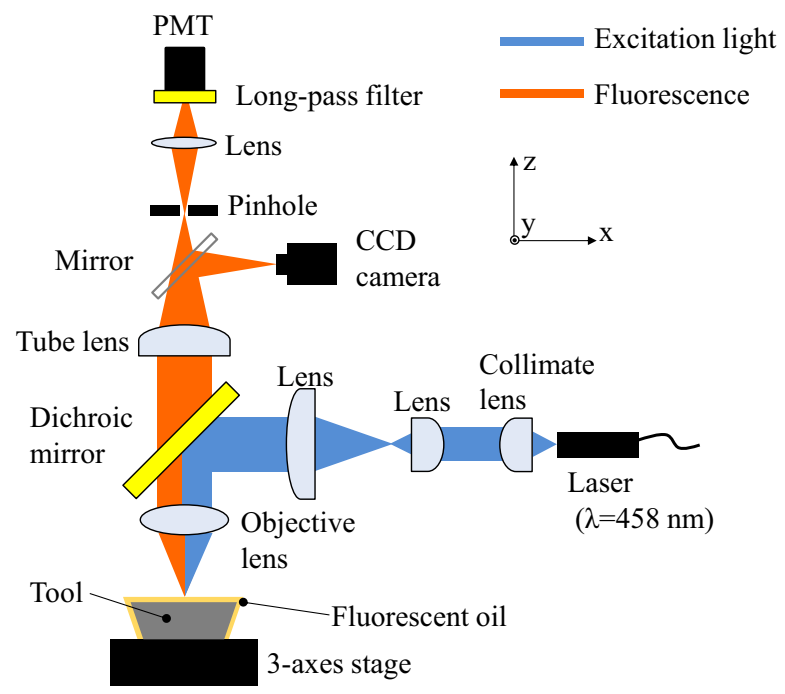

Figure 2 Schematic of optical system for fluorescent detection

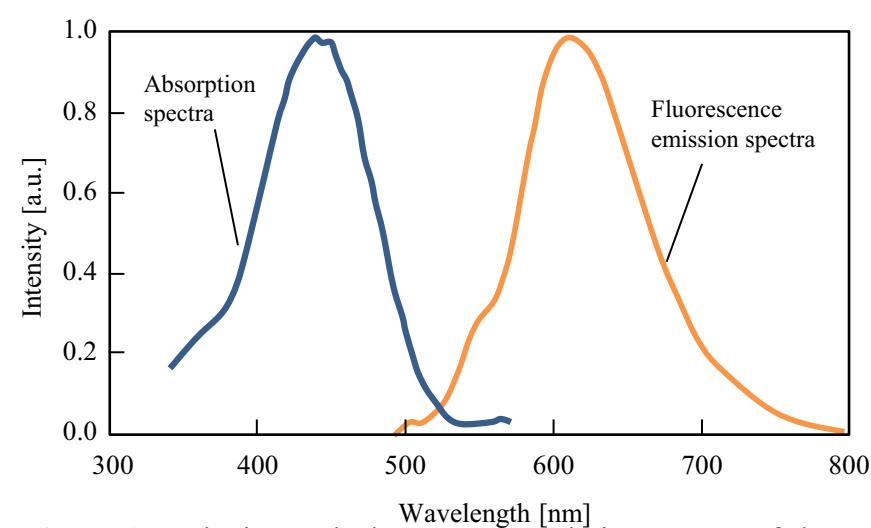

Figure 3 Excitation and Fluorescence emission spectra of the fluorescent dye used Fluorescent oil.

\section{Experimental results and discussion}

As a verification of the optical system, fluorescence emitted from horizontal and vertical surface is detected. By using silicon wafer that Fluorescent oil was thinly coated by spatula, fluorescence from horizontal and vertical surface is detected. 


\subsection{Fundamental verification of the optical system}

As shown in Fig. 4, the specimen coated Fluorescent oil is set horizontally against the object lens of optical system. The specimen is approached to the object lens along with $\mathrm{z}$-axes by moving the stage. Thus, a focus point of the object lens can through the layer of Fluorescent oil. The measured fluorersent signal is shown in Fig. 5, The data is normalized by maximum voltage of the detected signal. Stable signal can be seen from horizontal surface. The surface of silicon wafer seems to be in 20 to $30 \mu \mathrm{m}$ of $\mathrm{z}$-axes, since the signal is decreased.

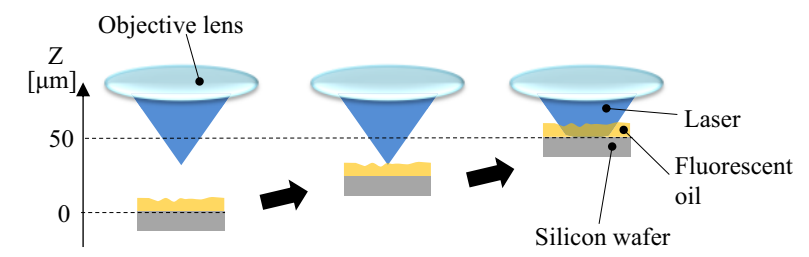

Figure 4 Detecting method of fluorescence from horizontal surface

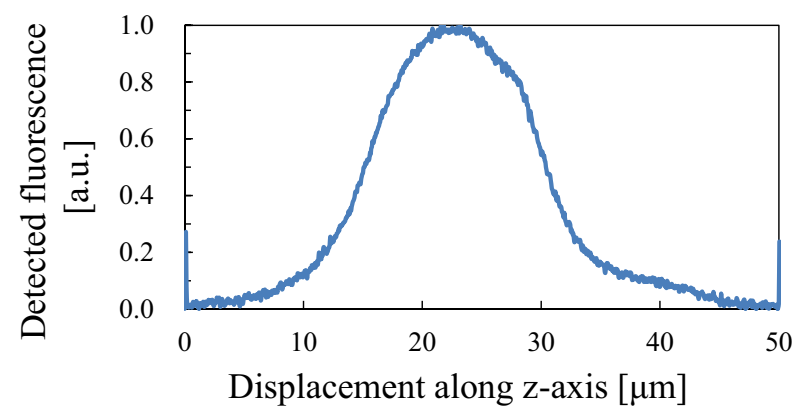

Figure 5 Detected fluorescence signal scanned against horizontal surface

As can be seen in Fig. 6, the specimen coated Fluorescent oil is set vertically against the object lens of optical system. The sample is scanned $50 \mu \mathrm{m}$ in the $\mathrm{x}$ direction, in order to allow Fluorescent oil to pass through the areas of focus position sufficiently. Fig. 7 shows the detected fluorescent signal of the experiment. The data is normalized by maximum voltage of the detected signal. It is also considered to succeed to detect fluorescence from the Fluorescent oil on the specimen. Moreover, since the decreased signal can be seen around the $\mathrm{z}$-direction scanning volume $28 \mu \mathrm{m}$, it is expected that the surface of the silicon wafer is present, if the oil thickness is enough thin. From this result, the proposed method enables to measure a vertical surface that is hard to measure with conventional optical methods.
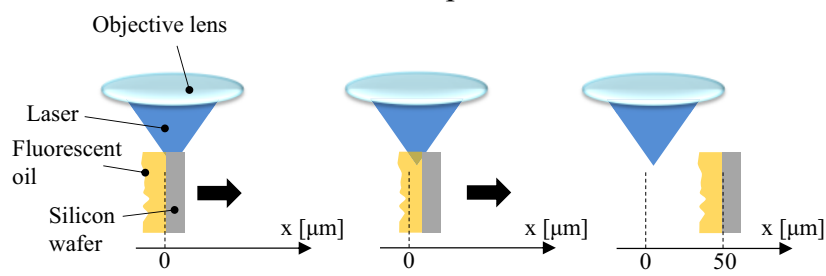

Figure 6 Detecting method of fluorescence from vertical surface

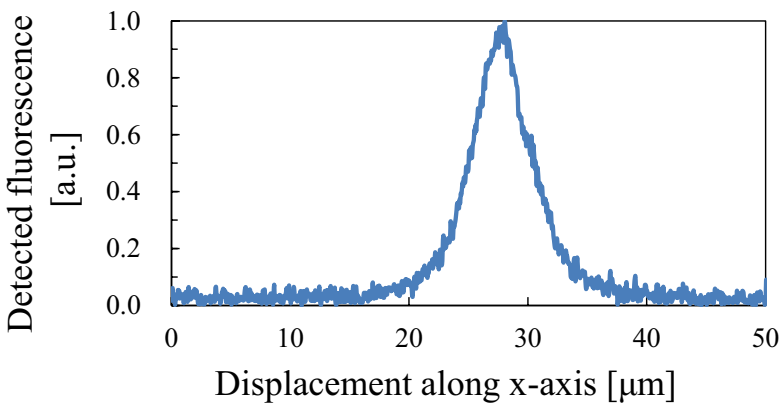

Figure 7 Detected fluorescence signal scanned against vertical surface

\subsection{Measurement of horizontal surface of cutting edge of a milling tool by detecting fluorescence}

To examine a possibility of the proposed method, an actual profile of cutting tool edge with wear is measured by the proposed method. Wear profile of a used milling cutting tool coated Fluorescent oil is measured with the optical system described in chapter 3. By comparing the measurement results with the results of the profilemeter (Kosaka Lab. Ltd., SE-3500K), it is examined whether the proposed method is feasible to measure the actual tool wear. Using Fluorescent oil, the cutting edge profile of the milling tool (SEKN42MT, Sumitomo Electric Hardmetal Corp.) with wear is measured.

A scheme of the measurement is shown in Fig. 8. Fluorescent oil is coated to the cutting edge of the milling tool as much as possible uniformly. The optical system scans $100 \mu \mathrm{m}$ in the $\mathrm{z}$-direction, and detects the fluorescence from Fluorescent oil as a signal. The stage of the optical system drives the tool $10 \mu \mathrm{m}$ step in the $\mathrm{x}$ direction to change the measuring point for each detection of the fluorescence signal again. A peak point of detected fluorescence signal is regarded as just a surface of the tool. By performing the same scanning, the wear of the shape of the region close to the horizontal plane of the chamfered face toward the rake face is measured. 2 areas of the cutting tool edge profile - worn deeply and worn shallowly, are measured by the above method 3 times. The same erea is measured by the profilemeter, and compares the both results.

Fig. 9 shows a example data of the detected fluorescence signal in this experiment. Red dots shown in Fig. 10 are measurement results by the proposed method. Black solid lines shown in Fig. 10 are measurement results by profile meter. Fig. 10 (a) to (c) show profiles of the milling tool edge after cutting carbon steel called "S53C" for $300 \mathrm{~m}$ at a cutting speed of $100 \mathrm{~m} / \mathrm{min}$. Fig. 10 (d) to (f) show profiles of the milling tool edge after cutting S53C for $100 \mathrm{~m}$ at a cutting speed of $400 \mathrm{~m} / \mathrm{min}$. Results from Fig. 10 (a) to (c) show that the features of the milling tool edge profiles measured by the proposed method are good agreement with the feature of profiles measured by profile meter. According to measurement results by profile meter, the average crater wear depth on $\mathrm{z}$-axis is about $2.0 \mu \mathrm{m}$, while the average crater wear depth results from the proposed method is about $1.4 \mu \mathrm{m}$. 
From those results, the proposed method makes it possible to measure the tool wear within several $\mu \mathrm{m}$ crater.

However, any measuring results of milling tool edge with deep crater wear shown in Fig. 10 (d) to (f), is out upward $10 \mu \mathrm{m}$ or more than the profiles measured by the profilemeter.

(a)

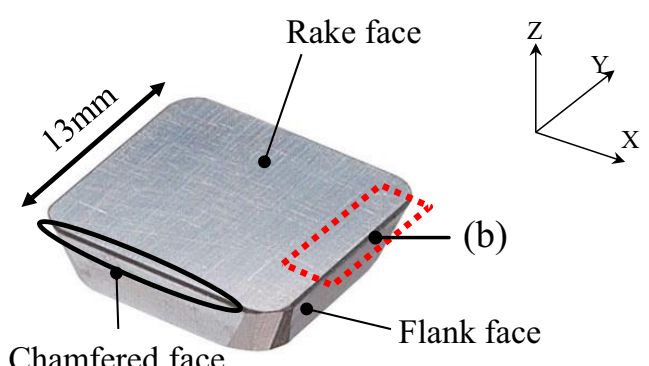

(b)

$$
\text { Excitation Fluorescent }
$$

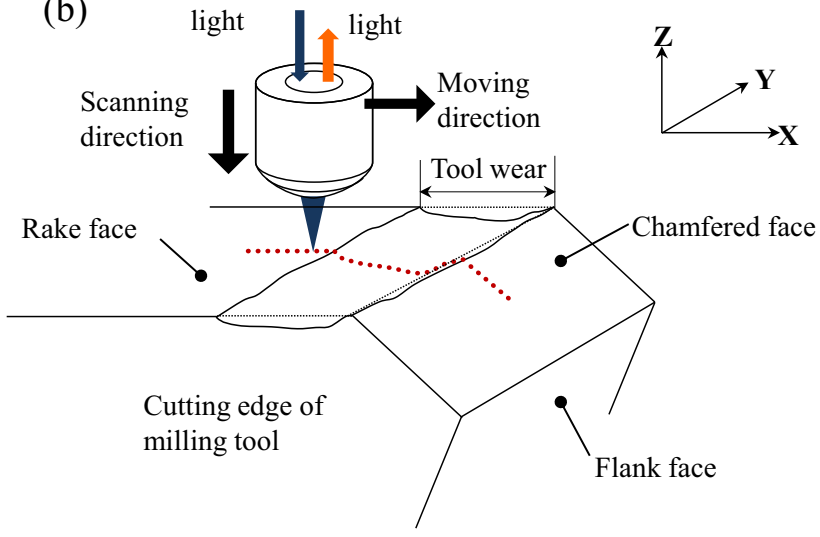

Figure 8 Measurement method of horizontal surface of cutting edge of milling tool. (a) milling tool to be measured, (b) magnification image of red dotted square in Fig. 7(a).

\subsection{Discussion}

As can be seen in Fig. 10(d) to (f), gap of profiles between the results by proposed method and the results by the profilemeter was occurred.

According to Fig. 3, the excitation wavelength of the optical system used in this paper $(458 \mathrm{~nm})$, is absorbed almost $100 \%$, while having ability to excite the fluorescence in high efficiency. In other words, the excitation light seems to be almost absorbed near the surface of Fluorescent oil, before reaching to deep point of Fluorescent oil or surface of the tool (Fig. 11(a)). Therefore, the fluorescent signal shown in Fig. 9 is considered to be a signal attenuated at the vicinity surface of Fluorescent oil, and attenuated point is erroneously regarded as the surface point of the tool. In the case of actual on-machine measurement, this result means that the tool surface sticking thick cutting fluid cannot be measured by the proposed method. In an actual measurement environment, there is cutting fluid with film thickness more than $10 \mu \mathrm{m}$ in the surface of cutting edge with wear [7]. Thus, the excitation light must permeate the thick oil film and reach the tool surface with exciting the oil.

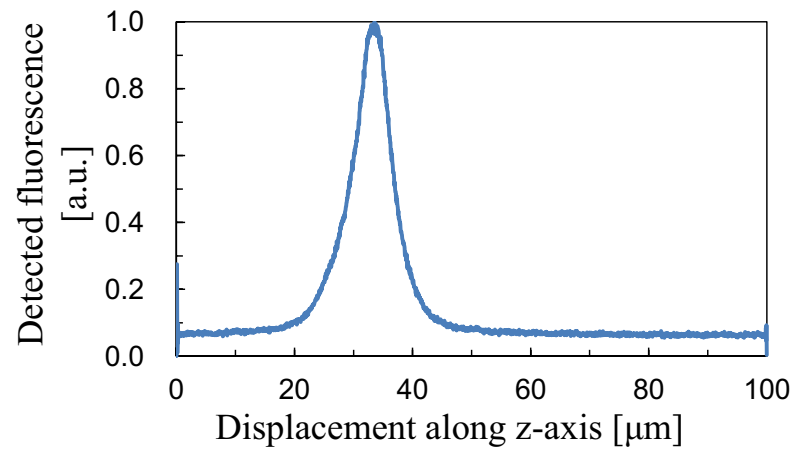

Figure 9 Example of detected Fluorescence signal from surface of the cutting edge of milling tool
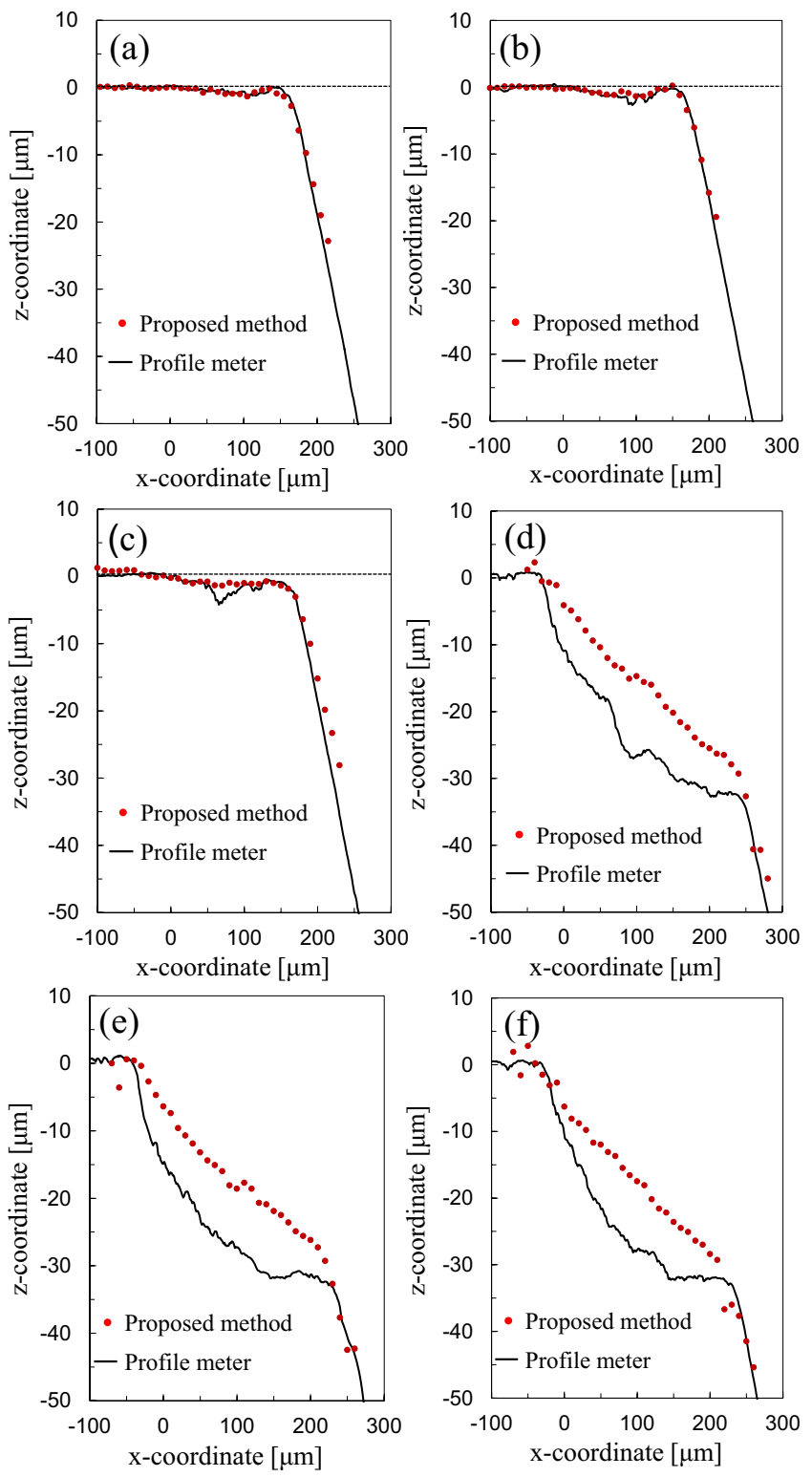

Figure 10 Measurement results of cutting edge of milling tool by proposed method and by profile meter. (a)-(c) after cutting S53C for $300 \mathrm{~m}$ at a cutting speed of $100 \mathrm{~m} / \mathrm{min}$, (d)-(f) after cutting S53C for $100 \mathrm{~m}$ at a cutting speed of $400 \mathrm{~m} / \mathrm{min}$. 
Based on the above, we propose the solution to use the excitation light with a wavelength of around $500 \mathrm{~nm}$ for Fluorescent oil. In this case, the excitation light can excite the fluorescence through lower emitting rate in $20 \%$. Furthermore, since the wavelength of this excitation light is transmitted through the Fluorescent oil film, this excitation light has possibillity to reach the surface of the tool. Thus, the excitation light having wavelength of aroud $500 \mathrm{~nm}$ can pass through the thick film of Fluorescent oil with exciting Fluorescent oil. As a result, the surface profile of cutting tool edge is possible to be measured correctly as shown in Fig. 11(b).

\section{Conclusions}

In this paper, we proposed the novel on-machine cutting tool edge profile measurement method detecting fluorescence emitted from cutting fluid adhered on surface of the tool. To verify the principle of the measurement, the fluorescent confocal microspocopy was constructed, and tried to detect fluorescence signals from horizontal and vertical surface of silicon wafer using Fluorescent oil. As results, fluorescence signals from horizontal and vertical surface were both detected. Since the proposed method enables to detect the signal from vertical surface that is hard to detect signal by conventional optical methods, this method has possibility to measure a cutting tool edge profile having complex structured such a vertical surface.

Furthermore, as a basic verification, cutting edge profile of milling tool with wear was measured with the proposed method by using Fluorescent oil. Then, a result of the cutting tool edge profile worn several $\mu \mathrm{m}$ was in a good agreement with the measurement result by Profile meter. However, profile measurement results worn over $10 \mu \mathrm{m}$ was shifted upward from the results of Profile meter. This gap seems to be caused because the excitation light was almost absorbed in the middle of Fluorescent oil film, before reaching the surface of the tool in the area with deep wear that Fluorescent oil is accumulated and forming a thick oil film.

Therefore, to estimate the correct profile by the proposed method, wavelength of the excitation light should be tuned according to absorbance and fluorescent properties of the fluorescent material. In this way, the excitation light is reachable at the surface of a tool. Hence, the fluorescent dye or the excitation light source should be changed after this.

\section{Acknowledgement}

We would like to thank Professor Mataka and Mr. Yazumi from International Science Technology., co ltd, Japan for providing the fluorescent dye. And we would like to thank Doctor T.Sugihara of Department of Mechanical Engineering, Osaka University, Japan, for a great support to provide milling tool and invaluable assistance and advice.

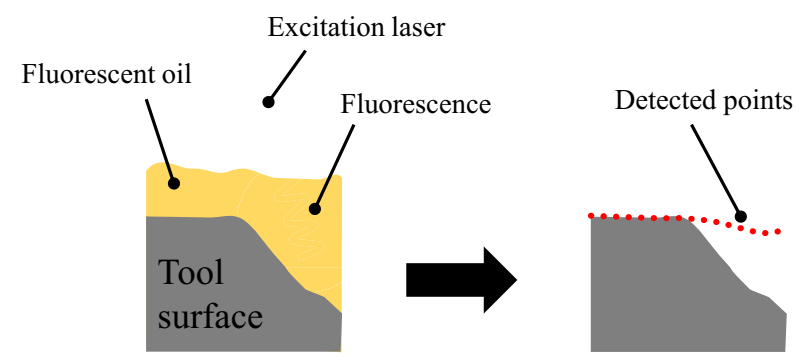

(a) Before optimizing the wavelength of excitation laser

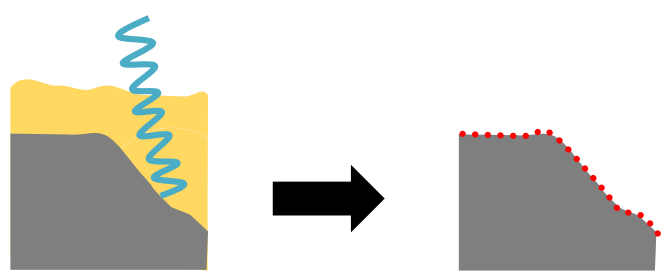

(b) After optimizing the wavelength of excitation laser

Figure 11 Relationship diagrams between fluorescent property and measurement results. (a) before optimizing a wavelength of excitation laser, (b) after optimizing the wavelength.

\section{References}

1. Nouari, M., and A. Ginting. "Wear characteristics and performance of multi-layer CVD-coated alloyed carbide tool in dry end milling of titanium alloy." Surface and Coatings Technology 200.18 (2006): 5663-5676.

2. W. Gao, et.al. : Precision and fast measurement of 3D cutting edge profiles of single point diamond micro-tools, CIRP Annals - Manufacturing Technology, 58/1 (2009) 451-454.

3. P. Khajornrungruang, et.al. : High precision tool cutting edge monitoring using laser diffraction for on-machine measurement, International journal of automation technology, 6, 2(2012) 163-167.

4. S.H. Jang, et.al. : A micro optical probe for edge contour evaluation of diamond cutting tools, Journal of sensors and sensor systems, 3 (2014), 69-76.

5. Rob Morris, et.al. : Environmental Spectroscopy Brings the Measurement to the Sample, International Environmental Technology, Nov. / Dec. (2013), 12-13.

6. M. Michihata, et.al. : Sensing a vertical surface by measuring a fluorescence signal using a confocal optical system, Meas. Sci. technol., 25, 6 (2014) 064004

7. G. Brandt, et.al. : Flank and crater wear mechanisms of alumina-based cutting tools when machining steel, Wear 112.1 (1986): 39-56. 